\title{
Unitarity potentials and neutron matter at the unitary limit
}

\author{
Huan Dong, L.-W. Siu and T. T. S. Kuo \\ Department of Physics and Astronomy, Stony Brook University, New York 11794-3800, USA \\ R. Machleidt \\ Department of Physics, University of Idaho, Moscow, Idaho 83844, USA
}

(Dated:)

\begin{abstract}
We study the equation of state of neutron matter using a family of unitarity potentials all of which are constructed to have infinite ${ }^{1} S_{0}$ scattering lengths $a_{s}$. For such system, a quantity of much interest is the ratio $\xi=E_{0} / E_{0}^{\text {free }}$ where $E_{0}$ is the true ground-state energy of the system, and $E_{0}^{f r e e}$ is that for the non-interacting system. In the limit of $a_{s} \rightarrow \pm \infty$, often referred to as the unitary limit, this ratio is expected to approach a universal constant, namely $\xi \sim 0.44(1)$. In the present work we calculate this ratio $\xi$ using a family of hard-core square-well potentials whose $a_{s}$ can be exactly obtained, thus enabling us to have many potentials of different ranges and strengths, all with infinite $a_{s}$. We have also calculated $\xi$ using a unitarity CDBonn potential obtained by slightly scaling its meson parameters. The ratios $\xi$ given by these different unitarity potentials are all close to each other and also remarkably close to 0.44 , suggesting that the above ratio $\xi$ is indifferent to the details of the underlying interactions as long as they have infinite scattering length. A sum-rule and scaling constraint for the renormalized low-momentum interaction in neutron matter at the unitary limit is studied. The importance of pairing in our all-order ring diagram and model-space HF calculations of neutron matter is discussed.
\end{abstract}

PACS numbers: pacs

\section{INTRODUCTION}

The 'unitary limit' of an ultracold Fermi gas refers to the special scenario where the inter-atomic interaction is tuned to have its scattering length $a_{s}$ approaching infinity $\left(a_{s}>>k_{F}^{-1}\right)$, leaving the Fermi momentum $k_{F}$ as the only relevant length scale in the many-body system. This limit was discussed as early as 1999 by Bertsch [1], who challenged many-body theorists with the question "What are the ground state properties of neutron matter, interacting with an infinite scattering length?". Under such condition the Fermions are strongly interacting, thus the determination of the ground state is highly non-trivial. However, the equation of state at this limit is expected to be an universal expression $E_{0}=\xi E_{0}^{\text {free }}$ where $\xi$ is an universal constant for any underlying Fermion systems. It is of much interest to determine $\xi$ experimentally or derive it theoretically, many such attempts having been made [2 27]. By far, the best numeric estimate on $\xi$ is considered to be $\xi=0.44(1)$ [9], 0.42(1) [13] and $0.40(1)$ [23] according to quantum Monte Carlo methods. The scattering length of trapped ultracold Fermi gases can in fact be magnetically tuned [29] by way of the Feshbach resonance. This is indeed a very important achievement, and it is based on this that the above constant $\xi$ for trapped ultracold Fermi gases can be experimentally measured. Several recently reported experimental values for $\xi$ are $0.46 \pm 0.05$ [4], $0.46_{-0.05}^{+0.12}$ [5] and $0.39(2)$ to $0.435(15)$ [6]

In a previous work 21], we have calculated the constant $\xi$ for neutron matter using a tuned CDBonn realistic nucleon-nucleon (NN) potential [30]. Unlike the case of trapped Fermion gases, here the scattering length of NN interactions still can not be tuned experimentally.
Thus we have chosen to tune the NN interaction by adjusting its meson parameters, in line with the Brown-Rho scaling [34] that the meson masses in nuclear medium is suppressed compared with their in-vacuum values. The ${ }^{1} S_{0}$ scattering length of the original CDBonn potential is $a_{s}=-18.9 \mathrm{fm}$, which is already fairly long. We have found that to have a very large scattering length (such as -12070 $\mathrm{fm}$ ) the meson parameters of the CDBonn potential need to be adjusted only slightly (about 2\%) [21]. This tuned CDBonn potential actually has given $\xi$ quite close to 0.44 for a wide range of densities $\left(\sim 0.02\right.$ to $\left.\sim 0.09 \mathrm{fm}^{-3}\right)[21$.

In the present work, we would like to investigate mainly the following question: Is the constant $\xi$ given by other unitarity potentials, defined as those with infinite scattering lengths, also close to 0.44 ? This constant is supposed to be an universal constant, and then its value should be independent of the detail structure of the potentials as long as they have infinite scattering length. In other words, $\xi$ given by all other unitarity potentials should in principle be the same. It may be difficult to prove this analytically. Before one can do so, it should be useful and of interest to first check this universality property numerically as we shall do in the present work. As it is rather involved to tune the various realistic NN potentials 31 33] to infinite scattering length, we have chosen to calculate $\xi$ from a family of simple hard-core squarewell (HCSW) potentials

$$
\begin{aligned}
V(r)= & V_{c} ; \quad r<r_{c} \\
& V_{b} ; \quad r_{c}<r<r_{b} \\
& 0 ; \quad r>r_{b} .
\end{aligned}
$$

An advantage of this type of potentials is that their scattering lengths can be given analytically and thus exact 
unitarity potentials can be readily obtained. The above HCSW potentials are clearly very different from the realistic NN potentials, yet as we shall discuss later the constant given by various HCSW potentials are all amazingly close to 0.44 , as is also given by the tuned CDBonn potential.

In the following we shall present some details of our calculations. In section IIA, we shall first briefly outline the renormalized low-momentum interaction $V_{\text {low }-k} 35$ 40] on which our calculations will be based. We shall carry out calculations using both HCSW and CDBonn potentials, both having strong repulsive cores. As is wellknown, these hard-core potentials are not suitable for being used directly in many-body calculations. In contrast, the low-momentum interaction $V_{\text {low }-k}$ is a smooth and energy-independent potential, and it has been extensively applied to calculations of nuclear matter [41], nuclear structure [44] and neutron star [45]. This interaction is obtained using a renormalization group ( $R G$ ) method where the high-momentum components of an underlying potential beyond a decimation scale $\Lambda$ are integrated out. The low-energy phase shifts are preserved by the $\mathrm{RG}$ procedure and so is the scattering length. In section IIB the procedures for constructing our model unitarity potentials are presented. Analytical expressions for the scattering length of HCSW potentials are given. From these expressions, one can construct an unlimited number of unitarity potentials (which all have infinite scattering length).

In section IIC, we shall describe how we calculate the neutron matter equation of state (EOS) from the low-momentum interaction $V_{\text {low-k }}$. Two methods will be employed: a ring-diagram method [21, 41] and a model-space Hartree-Fock (MSHF) method. There have been several many-body methods for calculating the EOS for large (number of particles approaching infinity) Fermionic systems such as atomic gases and nuclear matter. For a long time, the Brueckner-Hartree-Fock (BHF) theory 46 48 was the commonly used framework for such EOS calculations; an inconvenience of this method is that the BHF $G$-matrix interaction is energy dependent, adding numerical complications. We shall use the energy independent $V_{\text {low }-k}$ interaction mentioned earlier for the EOS calculations, to circumvent the above inconvenience. With the use of the $V_{l o w-k}$ interaction, the MSHF method provides a simple way for calculating the constant $\xi$. In fact MSHF is rather similar to BHF: In BHF the particle-particle correlations above $k_{F}$ are incorporated by way of the energy-dependent $G$-matrix, while in MBHF they are included via the energy-independent $V_{\text {low }-k}$ interaction. As we shall discuss later, the constant $\xi$ given by the ring-diagram method and the MSHF method are in fact nearly identical (both close to 0.44). We shall also discuss that the MSHF mean field potential should obey certain special constraint at the unitary limit. The importance of pairing in fermionic systems such as neutron matter has been well known and extensively studied (see e.g. [24, 27] and references quoted therin). In section IIC, we shall discuss that pairing has also played an important role in our all-order ring diagram calculations where a pair of fermions are allowed to interact any number of times, forming a quasi-boson composed of a coherent pair of fermions. Our results will be presented and discussed in section III. A summary and conclusion will be given in section IV.

\section{FORMALISM}

\section{A. Low-momentum interactions}

Since our calculations are largely dependent on the recently developed low-momentum interaction $V_{l o w-k}$, it may be useful to present first a short review on this interaction. As we know, the strong short-range repulsion contained in $V_{N N}$ usually requires a special renormalization treatment before being used in many-body calculations. A familiar such treatment is the BHF $G$-matrix method where the $G$-matrix interaction is obtained by summing the particle-particle ladder diagrams to all orders [46 -48]. An inconvenient aspect of this method is the energy dependence of $G$, making the many-body calculations based on $G$ rather complicated. In the past several years, there has been much progress in the $\mathrm{RG}$ approach to the NN interaction. A central idea here is that to describe the low-energy properties of a physical system it should be adequate to employ only a low-momentum effective interaction confined within a momentum decimation scale $\Lambda . V_{\text {low }-k}$ is such a low-momentum effective interaction. Starting from a realistic NN potential $V_{N N}$, $V_{l o w-k}$ is obtained from the following $T$-matrix equivalence equations [35-40]: The half-on-shell $T$-matrix for the full-space interaction is

$$
\begin{aligned}
T\left(k^{\prime}, k, k^{2}\right) & =V_{N N}\left(k^{\prime}, k\right) \\
& +\frac{2}{\pi} \mathcal{P} \int_{0}^{\infty} \frac{V_{N N}\left(k^{\prime}, q\right) T\left(q, k, k^{2}\right)}{k^{2}-q^{2}} q^{2} d q,
\end{aligned}
$$

where $\mathcal{P}$ denotes principal-value integration and the intermediate state momentum $q$ is integrated from 0 to $\infty$ covering the whole space. Then we define a lowmomentum half-on-shell $T$-matrix by

$$
\begin{aligned}
& T_{\text {low }-k}\left(k^{\prime}, k, k^{2}\right)=V_{\text {low }-k}\left(k^{\prime}, k\right) \\
& \quad+\frac{2}{\pi} \mathcal{P} \int_{0}^{\Lambda} \frac{V_{\text {low }-k}\left(k^{\prime}, q\right) T_{\text {low }-k}\left(q, k, k^{2}\right)}{k^{2}-q^{2}} q^{2} d q
\end{aligned}
$$

where the intermediate state momentum is integrated from 0 to $\Lambda$, the model space cut-off. The low momentum $V_{\text {low }-k}$ is then obtained by requiring the equivalence of $T$-matrix in the model space,

$$
T\left(k^{\prime}, k, k^{2}\right)=T_{\text {low-k }}\left(k^{\prime}, k, k^{2}\right) ;\left(k^{\prime}, k\right) \leq \Lambda .
$$

The low-momentum interaction $V_{l o w-k}$ is obtained by solving the above three equations. Since this procedure preserves the half-on-shell $T$-matrix, it certainly 
preserves the low-energy $\left(<\Lambda^{2}\right)$ phase shifts and scattering length $a_{s}$ of $V_{N N}$. This ensures that if $V_{N N}$ is tuned to be a unitarity potential $\left(a_{s} \rightarrow \pm \infty\right)$, so is the corresponding $V_{\text {low }-k}$.

\section{B. Hard-core square-well unitarity potentials}

There are several realistic NN potentials (CDBonn 30], Argonne 31], Nijmegen [32], Chiral 33]) which all describe the two-nucleon low-energy experimental data very accurately. It would be of interest to obtain unitarity potentials from them by tunning their interaction parameters slightly. But technically this is not easy to carry out. So far only the CDBonn potential has been tuned to attain this limit $\left(a_{s}=-12070 \mathrm{fm}\right)$ 21]. In the present work, we choose hard-core square-well (HCSW) potentials as given by Eq.(1) for further studying the properties of neutron matter at unitary limit. An advantage of using them is that their scattering length $a_{s}$ can be analytically obtained, allowing us to study the properties of neutron matter using many HCSW potentials with any chosen scattering lengths (including infinity).

As indicated in Eq.(1), the HCSW potential is characterized by the height $V_{c}$ of the repulsive core, the depth $V_{b}$ of its attractive well, and the respective ranges $r_{c}$ and $r_{b}$. In the present work, we consider neutron matter with interactions only in the ${ }^{1} S_{0}$ channel, whose phase shift $\delta$ is readily obtained from Eq.(5) and (6). Namely

$$
\begin{array}{r}
\tan \left(\delta+K_{3} r_{b}\right)=\frac{K_{3}}{K_{2}} \tan \left(K_{2} r_{b}+\alpha\right) \\
\tan \left(\alpha+K_{2} r_{c}\right)=\frac{K_{2}}{K_{1}} \tanh \left(K_{1} r_{c}\right)
\end{array}
$$

with

$$
\begin{array}{r}
K_{1}=\sqrt{\left(V_{c}-E\right) \frac{m}{\hbar^{2}}}, \\
K_{2}=\sqrt{\left(E-V_{b}\right) \frac{m}{\hbar^{2}}}, \\
K_{3}=\sqrt{E \frac{m}{\hbar^{2}}},
\end{array}
$$

where $E$ is the scattering energy in the center-of-mass frame. From the above results, the scattering length $a_{s}$ is obtained from a low energy expansion of $k \cot \delta$ as

$$
a_{s}=-\frac{B}{A}
$$

with

$$
\begin{aligned}
A= & K_{10} K_{20}-K_{20}^{2} \tanh \left(K_{10} r_{c}\right) \tan \left[K_{20}\left(r_{b}-r_{c}\right)\right], \\
B= & K_{20} \tanh \left(K_{10} r_{c}\right)+K_{10} \tan \left[K_{20}\left(r_{b}-r_{c}\right)\right]-r_{b} K_{10} K_{20} \\
& +r_{b} K_{20}^{2} \tanh \left(K_{10} r_{c}\right) \tan \left[K_{20}\left(r_{b}-r_{c}\right)\right]
\end{aligned}
$$

where

$$
K_{10}=\sqrt{V_{c} \frac{m}{\hbar^{2}}}, \quad K_{20}=\sqrt{-V_{b} \frac{m}{\hbar^{2}}}
$$

TABLE I: Three different unitarity HCSW potentials.

\begin{tabular}{lcccccc}
\hline \hline Potentials & $V_{c}[\mathrm{MeV}]$ & $r_{c}[\mathrm{fm}]$ & $V_{b}[\mathrm{MeV}]$ & $r_{b}[\mathrm{fm}]$ & $a_{s}\left[\times 10^{6} \mathrm{fm}\right]$ & $r_{e}[\mathrm{fm}]$ \\
\hline HCSW01 & 3000 & 0.15 & -20 & 2.31 & 15.2 & 2.36 \\
HCSW02 & 3000 & 0.30 & -30 & 2.03 & 3.38 & 2.21 \\
HCSW03 & 3000 & 0.50 & -50 & 1.81 & -4.58 & 2.20 \\
\hline
\end{tabular}

The effective range $r_{e}$ for this potential can also be derived analytically; the result is not presented here because it is fairly lengthy.

Eq.(7) implies that the condition for being a unitarity potential (infinite scattering length) is $A=0$, namely

$$
r_{b}-r_{c}=\frac{1}{K_{20}} \tan ^{-1}\left[\frac{K_{10}}{K_{20} \tanh \left(K_{10} r_{c}\right)}\right] .
$$

It is well known that when the potential is tunned to the unitary limit (the Feshbach resonance), it has a bound state with its energy approaching to zero. It is readily checked that the condition for having such a bound state is the same as the $A=0$ one given above.

The above condition enables us to construct any number of unitarity potentials by varying any three of the four parameters $V_{c}, V_{b}, r_{c}$ and $r_{b}$. Some sample such potentials are listed in Table I. As seen, they all have very large (infinite) scattering lengths while the potentials themselves are significantly different from each other. For example, the depth of the attractive parts of them changes from -20 to $-50 \mathrm{MeV}$. The effective ranges of these potentials are also listed, all being close to the unitarityCDBonn's $r_{e}$ of $2.54 \mathrm{fm}^{-1}$. These potentials will be used to calculate the universal ratio $\xi \equiv E_{0} / E_{0}^{\text {free }}$ for neutron matter. As we shall soon discuss, the ratios $\xi$ given by these three largely different potentials are in fact nearly identical to each other.

\section{Ring-diagram and model-space Hartree-Fock methods}

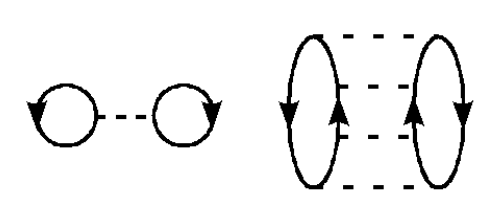

(a) (b)

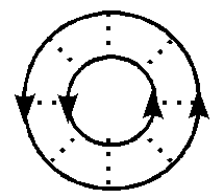

(c)
FIG. 1: Diagrams included in the all-order $p p h h$ ring-diagram summation for the ground state energy shift of nuclear matter. Each dashed line represents a $V_{l o w-k}$ vertex.

We use the ring-diagram method [21, 41] to calculate the neutron matter EOS. In this method, the groundstate energy shift $\Delta E$ is given by the all-order sum of the $p p h$ ring diagrams as illustrated in Fig.1, where (a), (b) and (c) are respectively 1st-, 4th- and 8th-order such 
diagrams. ( $\Delta E_{0}$ is defined as $\left(E_{0}-E_{0}^{\text {free }}\right)$ where $E_{0}$ is the true ground state energy and $E_{0}^{\text {free }}$ that for the non-interacting system.) In this way, we have

$$
\begin{aligned}
\Delta E_{0}=\int_{0}^{1} d \lambda \sum_{m} \sum_{i j k l<\Lambda} Y_{m}(i j, \lambda) & \\
& \times Y_{m}^{*}(k l, \lambda)\left\langle i j\left|V_{\text {low }-k}\right| k l\right\rangle,
\end{aligned}
$$

where the transition amplitudes are

$$
Y_{m}^{*}(k l, \lambda)=\left\langle\Psi_{m}(\lambda, A-2)\left|a_{l} a_{k}\right| \Psi_{0}(\lambda, A)\right\rangle,
$$

where $\Psi_{0}(\lambda, A)$ denotes the true ground state of nuclear matter which has $A$ particles while $\Psi_{m}(\lambda, A-2)$ the $m$ th true eigenstate of the $(A-2)$ system. Note that $\lambda$ is a strength parameter, integrated from 0 to 1 . Using the above $\Delta E_{0}$, the ratio $\xi$ is readily obtained, namely $\xi=1$ $+\Delta E_{0} / E_{0}^{\text {free }}$.

By summing the pphh ring diagrams to all orders, the above amplitudes $Y$ are given by the coupled equation of the form

$$
\begin{aligned}
A X+B Y & =\omega X \\
A^{*} Y+B^{*} X & =-\omega Y,
\end{aligned}
$$

where $X$ is $Y$ 's RPA partner. 42] $\omega$ is the excitation energy corresponding to either $\left(E_{m}(A-2)-E_{0}(A)\right)$ or $\left(E_{m}(A+2)-E_{0}(A)\right)$. It is readily recognized that the above RPA equation is of the same form as the wellknown quasi-boson RPA equations [43], resulting from treating the many-body system approximately as a collection of quasi bosons (each composed of a pair of interacting fermions). Thus our all-order ring-diagram calculation is actually a quasi-boson approximation for neutron matter, treating it as a system of quasi bosons. (This is not unexpected as RPA is a quasi-boson approximation.) Our all-order ring-diagram calculation is practically a pairing calculation in the sense that we include only the boson-forming ring diagrams to all orders (both forward and backward ladder diagrams for a pair of fermions interacting any number of times), allowing them to form a coherent pair (quasi-boson). Note, however, that our calculation becomes HF if we include only the 1st-order ring diagram, because in this case the above $Y$ amplitude becomes $Y(i j)=n_{i} n_{j}$ where $n_{i}=1$ for $i<k_{F}$ and $=0$ otherwise. Since we include only the ${ }^{1} S_{0}$ interaction, the quasi bosons in our present ring-diagram calculation are in fact all BCS-pairing bosons $\left({ }^{1} S_{0}\right)$.

The ring-diagram method described above is a modelspace formalism, where all nucleons are confined within a momentum model space $P(k<\Lambda)$, and the decimation scale for the $V_{\text {low }-k}$ interaction is the same $\Lambda$. The expression Eq.(10) for the energy shift $\Delta E_{0}$ is rather complicated, and is not convenient for studying the properties of the underlying interaction at the unitary limit. To have a clearer way to study these properties, we have considered a simpler method to calculate $\Delta E_{0}$, namely a model-space HF method (MSHF). In this method, we have

$$
\Delta E_{0}=\frac{1}{2} \Sigma_{k_{1}, k_{2} \leq k_{F}}\left\langle\vec{k}_{1} \vec{k}_{2}\left|V_{\text {low }-k}^{k_{F}}\right| \vec{k}_{1} \vec{k}_{2}\right\rangle .
$$

This is also a model-space approach where all nucleons are confined within a model space $P\left(k<k_{F}\right)$, but MSHF has a special feature that the interaction used is renormalized according to the same $P\left(k<k_{F}\right)$, namely the $V_{\text {low }-k}$ interaction is calculated with $\Lambda=k_{F}$ as denoted by $V_{l o w-k}^{k_{F}}$. A main difference between MSHF and the usual HF calculations is that the interaction used in the latter is not required to be renormalized according to the above model space while it is so for the former. In fact MBHF is equivalent to the ring-diagram method when one chooses $\Lambda$ to be its smallest value allowed by the Pauli principle, namely $k_{F}$. When $\Lambda=k_{F}$, diagrams like (b) and (c) of Fig.1 no longer exist (since particles with $k>k_{F}$ are disallowed in the model space); only diagram (a) remains, which is just MBHF. It may be mentioned that MBHF is rather similar to the familiar BHF method [46 48$]$. They both employ the same model space $P\left(k<k_{F}\right)$ (all nucleons confined within the Fermi sea), but they employ different model-space effective interactions. In BHF the energy-dependent $G$ matrix interaction is used while in MBHF the energyindependent $V_{\text {low }-k}$ interaction is employed; both include renormalizations from all-order particle-particle correlations beyond $k_{F}$, but for $G$ the renormalization is carried out using an energy-dependent method while an energyindependent one is applied for $V_{l o w-k}$. The MBHF ground-state energy is given by the sum of the kinetic energy and a simple integral, namely

$$
\begin{aligned}
\frac{E_{0}}{A}= & \frac{3}{5} \varepsilon_{F}+\frac{8}{\pi} \int_{0}^{k_{F}} k^{2} d k\left[1-\frac{3 k}{2 k_{F}}+\frac{k^{3}}{2 k_{F}^{3}}\right] \\
& \times \sum_{\alpha}\left(2 J_{\alpha}+1\right)\left\langle\alpha, k\left|V_{\text {low }-k}^{k_{F}}\right| \alpha, k\right\rangle .
\end{aligned}
$$

In the above $\varepsilon_{F}=\frac{\hbar^{2} k_{F}^{2}}{2 m}, k$ denotes the two-nucleon relative momentum and $J_{\alpha}$ is the total angular momentum for the partial wave $\alpha$. (We consider neutrons with interactions only in the ${ }^{1} S_{0}$ partial wave, and thus the above summation has only one term.) The above result may be useful for studying the properties of the $V_{\text {low }-k}$ interaction at the unitary limit. If the ratio $E_{0} / E_{0}^{\text {free }}$ is equal to a constant $\xi$ at this limit, then the above equation implies that $V_{\text {low-k }}^{k_{F}}$ at this limit must satisfy

$$
\begin{aligned}
\frac{3 \varepsilon_{F}}{5}(\xi-1)= & \frac{8}{\pi} \int_{0}^{k_{F}} k^{2} d k\left[1-\frac{3 k}{2 k_{F}}+\frac{k^{3}}{2 k_{F}^{3}}\right] \\
& \times\left\langle{ }^{1} S_{0}, k\left|V_{\text {low }-k}^{k_{F}}\right|^{1} S_{0}, k\right\rangle .
\end{aligned}
$$

This is a rather strong constraint for the interaction. Clearly there are many potentials which can satisfy the above constraint, allowing many potentials to have the same ratio $\xi$. In the following section, we shall discuss and numerically check this constraint. 
Within the above MSHF framework, the single-particle (s.p.) potential $U$ is given [42] as

$$
\begin{aligned}
U\left(k_{1}\right)= & \sum_{\alpha}\left(2 J_{\alpha}+1\right)\left\{\frac{16}{\pi} \int_{0}^{k_{-}} k^{2} d k\left\langle k \alpha\left|V_{\text {low }-k}^{k_{F}}\right| k \alpha\right\rangle\right. \\
& +\frac{2}{k_{1} \pi} \int_{k_{-}}^{k_{+}} k d k\left[k_{F}^{2}-k_{1}^{2}+4 k\left(k_{1}-k\right)\right] \\
& \left.\times\left\langle k \alpha\left|V_{\text {low-k }}^{k_{F}}\right| k \alpha\right\rangle\right\}
\end{aligned}
$$

with

$$
k_{-}=\left(k_{F}-k_{1}\right) / 2, \quad k_{+}=\left(k_{F}+k_{1}\right) / 2 .
$$

The MSHF s.p. spectrum is

$$
\varepsilon\left(k_{1}\right)=\frac{\hbar^{2} k_{1}^{2}}{2 m}+U\left(k_{1}\right)
$$

which can be well approximated by

$$
\varepsilon\left(k_{1}\right)=\frac{\hbar^{2} k_{1}^{2}}{2 m^{*}}+\Delta
$$

where $m^{*}$ is the effective neutron mass in medium and $\Delta$ is a constant related to the depth of the potential well. The MSHF ground-state energy can be expressed in terms of $m^{*}$ and $\Delta$. Then if this energy is equal to $\xi E_{0}^{\text {free }}$ at the unitary limit, the MSHF $m^{*}$ and $\Delta$ should satisfy the linear constraint

$$
\xi=\frac{1}{2}+\frac{m}{2 m^{*}}+\frac{5 \Delta}{6 \varepsilon_{F}} .
$$

In the following section, we shall also discuss and numerically check the above linear constraint concerning the MSHF sp potential.

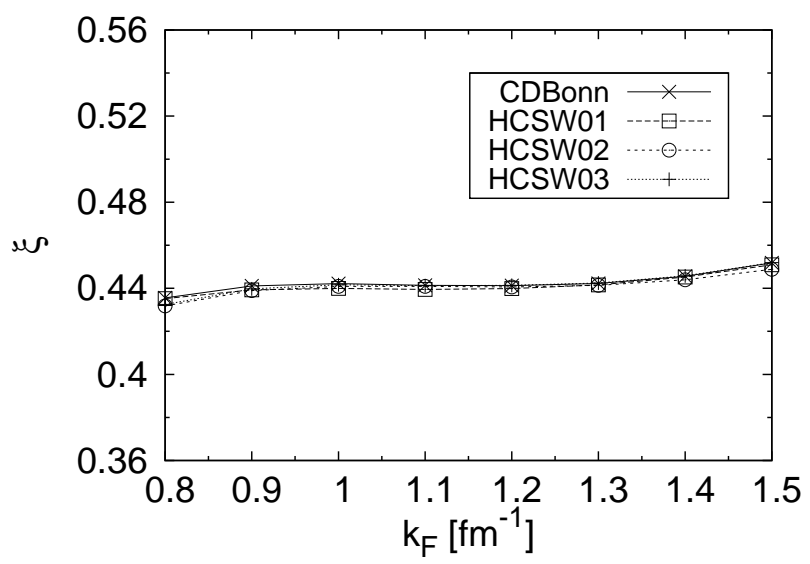

FIG. 2: Ring-diagram unitary ratios $\xi$ given by different unitarity potentials.

\section{RESULTS AND DISCUSSIONS}

We first calculate the ratio $\xi$ with the ring-diagram method (see Eq.(9)) using both the three HCSW unitarity potentials of Table I and the unitarity CDBonn potential $\left(a_{s}=-12070 \mathrm{fm}\right)$ 21]. As shown in Fig.2, the results given by these four potentials are all quite close to each other and to 0.44. A common decimation scale of $\Lambda=2.1$ $\mathrm{fm}^{-1}$ has been used for the results presented there. We have found that the $\xi$ values given by the ring-diagram calculations using the HCSW potentials are rather stable (variation less than $\sim 0.002$ ) with $\Lambda$ in the range between $\sim 2.0$ and $\sim 2.4 \mathrm{fm}^{-1}$. As found in 21], they are also quite stable for the unitarity CDBonn potential in the same range. Since these potentials have given nearly identical results for the ration $\xi$, it is of interest to compare their $V_{N N}(k, k)$ matrix elements from which the $\xi \mathrm{s}$ are calculated. Such a comparison is presented in Fig.3, and as seen they are actually quite different. It is indeed surprising that the ratios $\xi$ given by these vastly different interactions are nearly the same. To illustrate the key role of the unitary limit, we further calculate the ratios $\xi$ near the unitary limit as displayed in Fig.4. When $a_{s}$ is away from the unitary limit, the $\xi$ values given by these potentials are noticeably different, but they converge to a common value only as $1 / a_{s}$ approaching zero. Above results strongly suggest that the ratio $\xi$ is indifferent to the details of the underlying potentials, as long as they have infinite (or very large) scattering lengths, namely they are all unitarity potentials.

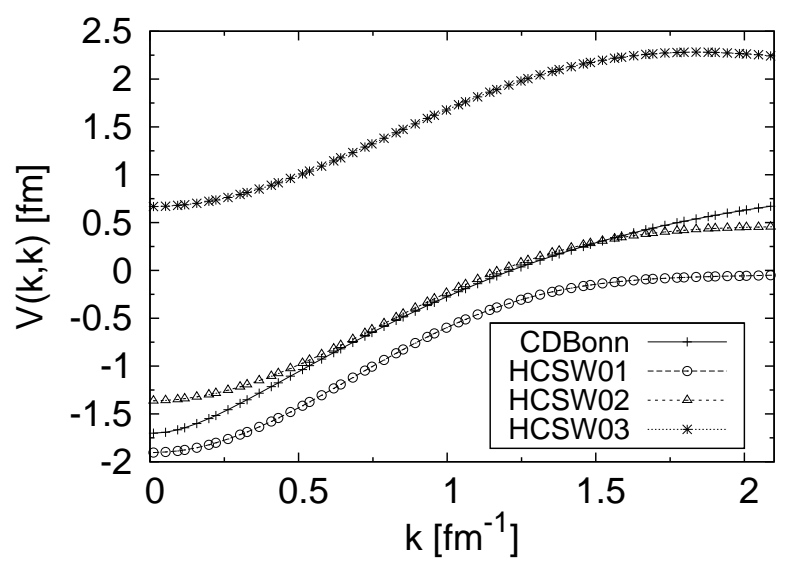

FIG. 3: Diagonal matrix elements $V_{N N}(k, k)$ of different unitarity potentials.

Using the above four unitarity potentials, we have also calculated the ratios $\xi$ using the MSHF method (see Eq.(14)). The results are presented in Fig.5, and as seen the results given by the four potentials are nearly identical and they are all quite close to 0.44 over a wide range of densities. It is of interest to note that the MSHF results shown are remarkably close to the ring-diagram ones of Fig.2. This close agreement is an indication that the hole- 


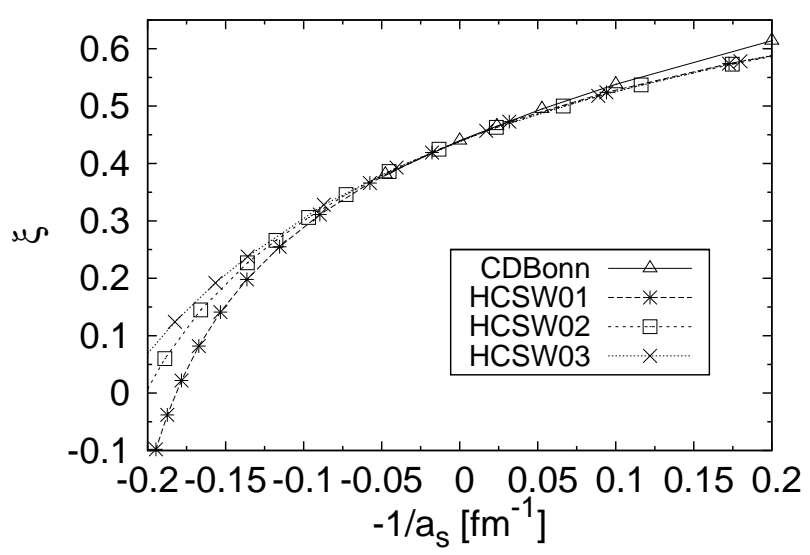

FIG. 4: The ring-diagram unitary ratios $\xi$ near the unitary limit. $k_{F}=1.2 \mathrm{fm}^{-1}$ is used.

hole correlation diagrams like diagram (c) of Fig.1 may not be important for pure neutron matter. The contribution from the particle-particle correlations like diagrams (a) and (b) is included in both ring diagram and MSHF calculations, but diagrams like (c) are included only in the former. It may be noted that in both Fig.2 and Fig.5 our results are nearly constant for $k_{F}$ between $\sim 0.9$ and $\sim 1.4 \mathrm{fm}^{-1}$ (corresponding to density range $\sim 0.016$ to $\sim 0.092 \mathrm{fm}^{-3}$ ). Outside this range, small variations of $\xi$ start to appear. These variations are an indication that the methods we have employed for the calculation is not adequate for the high and very low density regions indicated above. So far we have employed only two-body $V_{\text {low }-k}$ interactions in the calculation. Three body interactions have not been considered in the present work, and their effects may be important. Further studies in this direction are needed.

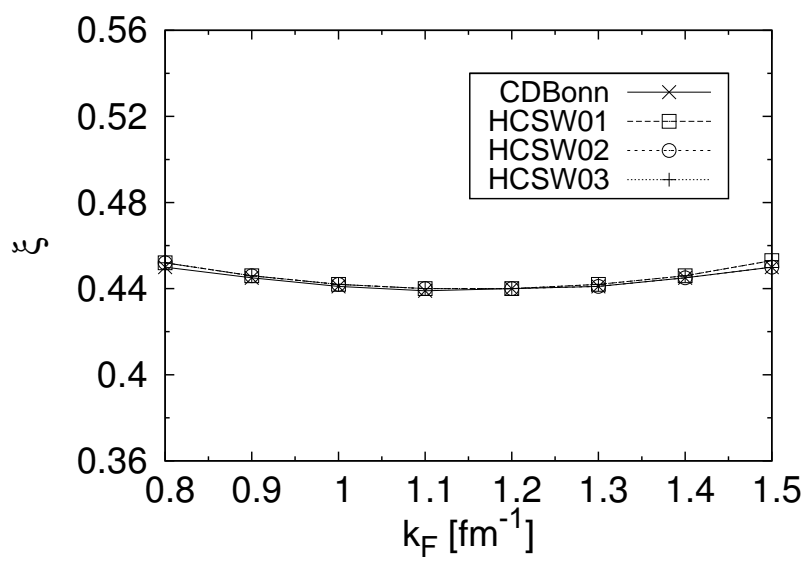

FIG. 5: MSHF unitary ratios $\xi$ given by different unitarity potentials.

In the MSHF method, the ratio $\xi$ is calculated from the model-space effective interaction $V_{\text {low-k }}^{k_{F}}$. We have seen from Fig. 5 that the ratios $\xi$ given by the four dif-

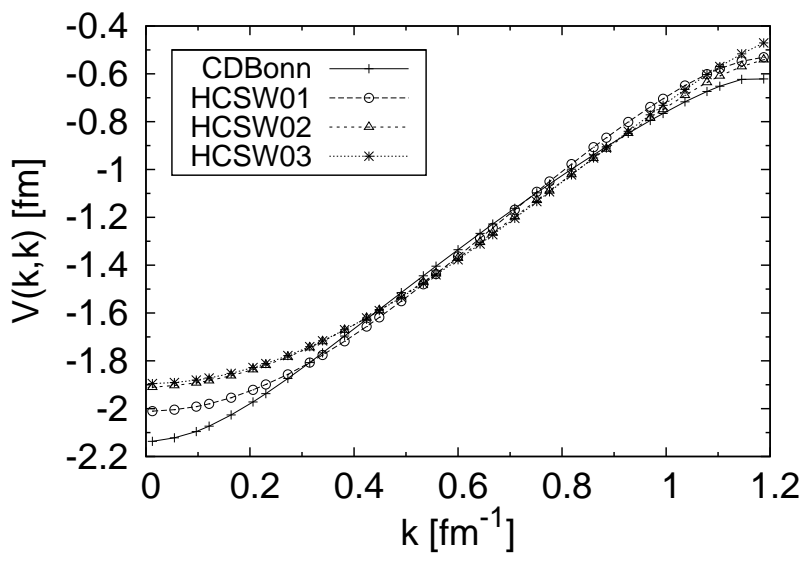

FIG. 6: Diagonal matrix elements of $V_{l o w-k}^{k_{F}}$ from different unitarity potentials. $\Lambda=k_{F}=1.2 \mathrm{fm}^{-1}$ is used.

ferent potentials are nearly the same. Does this mean that their $V_{\text {low }-k}^{k_{F}}$ are also nearly the same? In Fig.6 the matrix elements of this interaction for four different unitarity potentials are presented. As seen, they are clearly not identical to each other; there are significant differences among them. Eq.(14) is in fact a strong constraint for the above interactions. To have $\xi$ being a universal constant, the left-hand side of Eq.(14) is proportional to $k_{F}^{2}$. Consequently the integral in the right-hand side must be equal to $C \times k_{F}^{2}, C$ being a constant, noting that its integrand and integration limit are both dependent on $k_{F}$. This implies that at the unitary limit the $V_{l o w-k}^{k_{F}}$ interaction must satisfy certain stringent requirements so as to satisfy the above constraint.

Similar to the effective interactions commonly used in effective field theories [28, 49], we have found that our $V_{\text {low }-k}^{k_{F}}$ interactions can be highly accurately simulated by low-order momentum expansions of the form

$$
\left\langle k\left|V_{\text {low }-k}^{k_{F}}\right| k\right\rangle=V_{0}+V_{2}\left(\frac{k}{k_{F}}\right)^{2}+V_{4}\left(\frac{k}{k_{F}}\right)^{4},
$$

where $V_{0}, V_{2}$ and $V_{4}$ are constants (independent of $k$ but dependent on $k_{F}$ ). (The rms deviations for fitting the results of Fig. 6 by the above expansion are $\sim 2 \times 10^{-2}$ and $\sim 2 \times 10^{-3}$ respectively for the CDBonn and HCSW cases, the fitting being very good.) In terms of these constants, Eq.(14) assume a rather simple form, namely

$$
\frac{3 \pi}{10}(\xi-1)=k_{F}\left(\frac{V_{0}}{3}+\frac{V_{2}}{10}+\frac{3 V_{4}}{70}\right) .
$$

The above is an interesting sum-rule and scaling constraint, namely at the unitary limit the strength sum $\left(V_{0} / 3+V_{2} / 2+3 V_{4} / 70\right)$ is a constant for any $k_{F}$ (density) and, in addition, it scales with $1 / k_{F}$ (i.e. proportional to $\left.1 / k_{F}\right)$. In Table II we present some sample results, to check how well do they satisfy the above constraint. As seen our results satisfy this constraint very well. For each $k_{F}$, the values of the above sum given by the four different unitarity potentials are all quite close to each other. 
The values of this sum for different $k_{F}$ are different but they are all giving $\xi$ close to 0.44 , in close agreement with the $1 / k_{F}$ scaling.

TABLE II: Low-order momentum expansion of $V_{l o w-k}^{k_{F}}$. Listed are the coefficients $V_{0}, V_{2}$ and $V_{4}$ of Eq.(20), with the sum $\left(V_{0} / 3+V_{2} / 10+3 V_{4} / 70\right)$ denoted as Sum. Four unitarity potentials are used.

\begin{tabular}{ccccccc}
\hline \hline$V_{N N}$ & $k_{F}\left[\mathrm{fm}^{-1}\right]$ & $V_{0}[\mathrm{fm}]$ & $V_{2}[\mathrm{fm}]$ & $V_{4}[\mathrm{fm}]$ & Sum & $\xi$ \\
\hline CDBonn & 1.2 & -2.053 & 3.169 & -1.801 & -0.445 & 0.434 \\
HCSW01 & & -2.001 & 2.865 & -1.402 & -0.441 & 0.439 \\
HCSW02 & & -1.904 & 2.373 & -0.999 & -0.440 & 0.440 \\
HCSW03 & & -1.893 & 2.261 & -0.825 & -0.440 & 0.439 \\
HCSW01 & 1.0 & -2.102 & 2.202 & -1.070 & -0.526 & 0.442 \\
HCSW01 & 1.4 & -1.945 & 3.584 & -1.983 & -0.375 & 0.443 \\
\hline
\end{tabular}

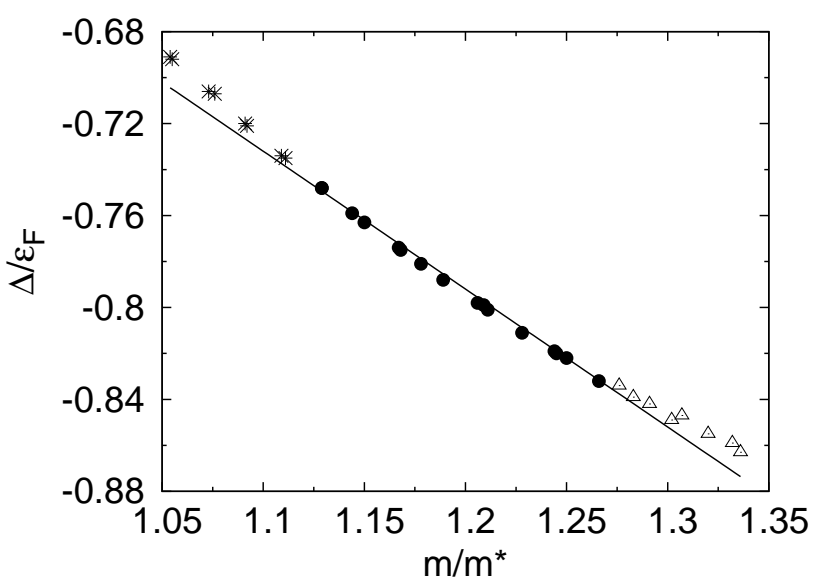

FIG. 7: MSHF effective mass $m^{*}$ and well depth $\Delta$ at the unitary limit. Results calculated from the unitarity potentials are denoted by 'star', 'dot' and 'triangle' respectively for $k_{F}$ in the ranges $(0.8-0.9),(0.9-1.4)$ and $(1.4-1.5) \mathrm{fm}^{-1}$. The solid line represents the linear expression Eq.(18) with $\xi=0.44$. See text for other explanations.

By far, the best numeric estimates of the ratio $\xi$ have been obtained from the variational quantum-MonteCarlo (QMC) calculations (see e.g. 24] and references quoted therein). It would be useful and of interest to compare our ring-diagram and MSHF calculations with the QMC ones. As reviewed in 24], superfluid-QMC calculations are generally based on the variation principle $\delta\left[\left\langle\Phi_{B C S}\left|\Psi_{J}^{\dagger} H \Psi_{J}\right| \Phi_{B C S}\right\rangle /\left\langle\Phi_{B C S}\left|\Psi_{J}^{\dagger} \Psi_{J}\right| \Phi_{B C S}\right\rangle\right]=0$, where $\Phi_{B C S}$ is a BCS-paired trial wave function of definite number of particles and $\Psi_{J}$ is a r-space Jastrow correlator whose inclusion depends on the interaction employed; its inclusion is necessary when an interaction with a 'hard-core' repulsion is employed 23 but not so for smooth potentials without 'hard core' 9, 11, 24]. Clearly pairing is incorporated in superfluid-QMC by using a BCS-paired trial wave function. As discussed in section II, we emphasize pairing differently, namely by including only the boson-forming ring diagrams to all orders (both forward and backward ladder diagrams for a pair of fermions interacting any number of times), and in this way our all-order ring-diagram calculation treats the system as a collection of quasi bosons. Despite this difference, it is encouraging that our result of $\xi \simeq 0.44$ is satisfactorily close to the superfluid-QMC value of $\sim 0.40$ to $\sim 0.44[23,24]$. It may be pointed out that when we include only the first order ring diagram, our ringdiagram calculation reduces to the usual HF calculation. For $\Lambda=2.1 \mathrm{fm}^{-1}$ and $k_{F}=0.8 \mathrm{fm}^{-1}$ we have carried out $\mathrm{HF}$ calculations (i.e. including only the 1st-order ring diagram) and obtained $\xi_{H F} \simeq 0.55$, for all the four unitarity potentilas mentioned earlier. This $\xi_{H F}$ is significantly larger than the corresponding all-order-ring result of 0.44. Normal-QMC calculations (where the trial wave function is a closed Fermi sea) have given $\xi \simeq 0.54$ 10, 24]. We believe that our 1st-order ring calculation is similar to that of the normal-QMC while our all-order ring-diagram one is similar to that of the superfluid-QMC (where the trial wave function is $\Phi_{B C S}$ ). Further studies of the above possible connections are needed.

We have discussed in section II that at the unitary limit the MSHF s.p. potential has a special property, namely its effective mass $m^{*}$ and well depth $\Delta$ satisfy a linear constraint dependent on the unitary ratio $\xi$ (see Eq.(18)). Here let us check how well is this constraint obeyed by our results. Similar to Fig.5, we have calculated $\mathrm{m} / \mathrm{m}^{*}$ and $\Delta / \varepsilon_{F}$ using the CDBonn and HCSW unitarity potentials using a wide range of $k_{F}$ from 0.8 to $1.5 \mathrm{fm}^{-1}$. Our results are plotted in Fig.7, and as seen most of our data are located near the straight line corresponding to $\xi=0.44$. In the figure, the results for $k_{F}$ in the range of $(0.9-$ 1.4) $\mathrm{fm}^{-1}$ are quite close to the $\xi=0.44$ straight line. For $k_{F}$ outside this range, they are slightly off the line, which is consistent with the results of Fig. 5.

It is promising that at the unitary limit the above MSHF framework has given rather satisfactory results for the ratio $\xi(\sim 0.44)$, in close agreement with both the superfluid-QMC results 23, 24] and those from our allorder ring diagram calculations. In addition, this method is fairly simple and transparent; as indicated by Eq.(15) $\xi$ is given by a simple integral and the HF potentials at the unitary limit satisfy the constraint of Eq.(19). It may be mentioned, however, that this MSHF is only a limited effective theory; it is the $\Lambda=k_{F}$ limit of the ring-diagram formalism. In this limit the model space is one dimensional, and the model-space ground state wave function is $\left|k_{F}\right\rangle$, the closed Fermi sea. The BCS pairing gap $\Delta_{B C S}$ is an important quantity, but this information is not contained in the MSHF effective theory. $\Delta_{B C S}$ is the lower bound for the lowest excitation energy of the many-body system. MSHF, however, is a one-dimensional effective theory which can describe only the ground state of the system; it provides no information about excited states. The effective MSHF theory is not capable to provide information about $\Delta_{B C S}$, even though the MSHF wave function $\left|k_{F}\right\rangle$ is completely $(\vec{k} \uparrow,-\vec{k} \downarrow)$ paired. To calculate $\Delta_{B C S}$, one may need to employ the number-nonconserving Green's function framework 
[50, 51] which has been commonly used in supercoductivity (superfluidity) calculations. (The Green's function method used in the present work is based on a numberconserving formalism, and is not suitable for calculating $\Delta_{B C S}$.) Using several Skyrme effective interactions, Su. et al. [51] have performed such number-nonconserving BCS calculations for nuclear matter. We plan to repeat their BCS calculations using instead the RG $V_{l o w-k}$ interactions, and will report our results in a separate publication.

\section{SUMMARY AND CONCLUSION}

Using several different unitarity potentials, defined as having infinite (very large) scattering length $a_{s}$, we have calculated the ground-state energy ratio $\xi \equiv E_{0} / E_{0}^{\text {free }}$ for neutron matter over a wide range of densities. A main purpose of our study was to check if the ratios so obtained are 'universal' in the sense that they have a common value (or nearly so), independent of the details of the potentials as long as they have $a_{s} \rightarrow \pm \infty$. We have used four unitarity potentials: One is the unitarity CDBonn potential whose meson exchange parameters are slightly tuned so that its $a_{s}$ becomes very large (-12070 $\mathrm{fm})$. The other three are square-well 'box' potentials with both hard-core repulsion and exterior attraction. The $a_{s}$ of these box potentials can be obtained analytically, making it easy to construct many unitarity box potentials of different ranges and depths.

We have calculated $\xi$ using two methods: a ring-diagram method and a model-space Hartree-Fock (MSHF) method. An important step in both methods is the transformation of the unitarity potentials, which have strong short-range repulsions, into low-momentum interactions $V_{\text {low-k }}$ which are smooth potentials, convenient for many-body calculations. The transformation is carried out using a renormalization group method which preserves low-energy phase shifts and the scattering length.
We have found that the ratios $\xi$ given by the ring-diagram and MSHF methods are practically identical over a wide range of densities (from $\sim 0.02$ to $\sim 0.09 \mathrm{fm}^{-3}$ ). This is an indication that the particle-particle hole-hole correlations (as shown by diagram (c) of Fig.1) are not important for neutron matter. Such correlations are included in the ring-diagram method but not in MSHF. The effect of the particle-particle ladder correlations (as shown by diagram (b) of Fig.1) are included in both. The MSHF method is considerably simpler than the ring-diagram one. It may provide a promising method for neutron matter. Further study of this method for neutron matter may be useful and of interest.

The CDBonn and the three box unitarity potentials are very different from each other, but the ratios $\xi$ predicted by them using either of the above methods are all remarkably close to 0.44 , over a wide range of densities. This is indeed a rather interesting and surprising result, indicating that $\xi$ may be perceived a universal constant. For the MSHF case, this result is related to some special properties of the $V_{\text {low }-k}$ interaction at the unitary limit. We have found that the $V_{l o w-k}$ interaction for MSHF can be accurately simulated by a low-order momentum expansion of the form $\left[V_{0}+V_{2}\left(k / k_{F}\right)^{2}+V_{4}\left(k / k_{F}\right)^{4}\right]$, and at the unitary limit the strength sum $\left(V_{0} / 3+V_{2} / 10+3 V_{4} / 70\right)$ calculated from the above potentials satisfies the constraint of Eq.(20) very well, for a wide range of $k_{F}$. It is also found that at the unitary limit the parameters $m^{*}$ and $\Delta$ of the MSHF mean field potentials given by the above potentials all obey a linear constraint satisfactorily. In conclusion, we believe that our results provide strong numerical support to the conjecture that the ratio $\xi$ is universal.

Acknowledgement We thank G.E. Brown and E. Shuryak for many helpful discussions. This work is supported in part by the U.S. Department of Energy under Grant Nos. DE-FG02-88ER40388 and DE-FG0203ER41270 (R.M.), and the U.S. National Science Foundation under Grant No. PHY-0099444.
[1] R.F. Bishop, Int. J. Mod. Phys.B 15,iii(2001), "ManyBody Challenge Problem by G.F.Bertsch".

[2] K.M. O'Hara, S.L. Hemmer, M.E. Gehm, S.R. Granade, J.E. Thomas, Science 298, 2179 (2002).

[3] M.E. Gehm, S.L. Hemmer, S.R. Granade, K.M. O'Hara, J.E. Thomas, Phys. Rev. A 68, 011401(R) (2003).

[4] G.B. Patridge, Wenhui Li, R.I. Kamar, Yean-an Liao, R.G. Hulet, Science 311, 503(2006).

[5] J.T. Stewart, J.P. Gaebler, C.A. Regal, and D.S. Jin, Phys. Rev. Lett 97, 220406(2006).

[6] Le Luo, J.E. Thomas J. Low. Temp. Phys.(2009) 154: $1-29$.

[7] G.A. Baker,Jr.,Phys. Rev. C60,054311(1999).

[8] H. Heiselberg, Phys. Rev. A 63, 043606(2001).

[9] J. Carlson, S.-Y. Chang, V.R. Pandharipande and K.E. Schmidt, Phys. Rev. Lett, 91,050401(2003).
[10] J. Carlson, J. Morales, Jr., V. R. Pandharipande and D. G. Ravenhall, Phys. Rev. C 68, 025802 (2003)

[11] S.Y. Chang and V.R. Pandharipande, J. Carlson, K.E. Schmidt, Phys. Rev. A 70, 043602 (2004)

[12] A. Perali, P. Pieri, and G.C. Strinati, Phys. Rev. Lett. 93, 100404(2004).

[13] G.E. Astrakharchik, J. Boronat, J. Casulleras and S. Giorgini, Phys. Rev. Lett. 93,200404(2004).

[14] J. Carlson and S. Reddy, Phys. Rev. Lett. 95, 060401 (2005).

[15] A. Bulgac and G.F. Bertsch, Phys. Rev. Lett. 94, 070401 (2005).

[16] A. Schwenk and C.J. Pethick, Phys. Rev. Lett. 95, 160401(2005)

[17] Y. Nishida and D.T. Son, Phys. Rev. Lett. 97, 050403(2006). 
[18] D. Lee and T. Schäfer, Phys. Rev. C 73, 015202(2006)

[19] R. Haussmann, W. Rantner, S. Cerrito and W. Zwerger, Phys. Rev. A 75, 023610(2007).

[20] J.-W. Chen and E. Nakano, Phys. Rev. A 75, 043620 (2007).

[21] L.-W. Siu, T.T.S. Kuo, and R. Machleidt, Phys. Rev. C 77, 034001 (2008).

[22] B. Borasoy, E. Epelbaum, H. Krebs, D. Lee and U.-G. Meißner, Eur. Phys. J. A 35, 357 (2008).

[23] A. Gezerlis and J. Carlson, Phys. Rev. C 77, 032801(R) (2008).

[24] S. Giorgini, L. P. Pitaevskii and S. Stringari, Rev. Mod. Phys. 80, 1215 (2008).

[25] E. Epelbaum, H. Krebs, D. Lee and U.-G. Meißner, Eur. Phys. J. A 40, 199 (2009).

[26] T. Abe and R. Seki, Phys. Rev. C 79, 054003 (2009).

[27] T. Schaefer and D. Teany, Rep. Prog. Phys. 72, 126001 (2009).

[28] E. Epelbaoum, W. Gloeckle, A. Krueger and U. Meiszner, Nucl. Phys. A465, 413(1999).

[29] C.A. Regal and D.S. Jin, Phys. Rev. Lett. 90, 230404(2003); S. Jochim, M. Bartenstein, G. Hendl, J.H. Denschlag, R. Grimm, A. Mosk and M. Weidemüller, ibid. 89, 273202(2002); C.H. Schunck, M.W. Zwierlein, C.A. Stan, S.M.F. Raupach, W. Ketterle, A. Simoni, E. Tiesinga, C.J. Williams and P.S. Julienne, Phys. Rev. A 71, 045601 (2005)

[30] R. Machleidt, Phys. Rev. C 63024001 (2001).

[31] R.B. Wiringa, V.G.J. Stoks and R. Schiavilla, Phys. Rev. C 51, 38 (1995).

[32] V.G.J. Stoks, R.A.M. Klomp, C.P.F. Terheggen and J.J. de Swart, Phys. Rev. C 49, 2950 (1994).

[33] D.R. Entem, R. Machleidt, Phys. Rev. C 68, 041001(R) (2003).

[34] G.E. Brown and M. Rho, Phys. Rev. Lett. 66, 2720(1991).
[35] S.K. Bogner, T.T.S. Kuo, L. Coraggio, A. Covello, Nucl. Phys. A684, 432(2001).

[36] S.K. Bogner, T.T.S. Kuo, L. Coraggio, A. Covello and N. Itaco, Phys. Rev. C65, 051301(R)(2002).

[37] L. Coraggio, A. Covello, A. Gargano, N. Itaco, T.T.S. Kuo, D.R. Entem and R. Machleidt, Phys. Rev. C 66,021303(R) (2002).

[38] A. Schwenk, G.E. Brown and B. Friman, Nucl. Phys. A703, 745(2002).

[39] S.K. Bogner, T.T.S. Kuo and A. Schwenk, Phys. Rep. 386,1 (2003).

[40] J.D. Holt, T.T.S. Kuo and G.E. Brown, Phys. Rev. C 69,034329(2004).

[41] L.-W. Siu, J.W. Holt, T.T.S. Kuo and G.E. Brown, Phys. Rev. C79, 054004 (2009).

[42] H.Q. Song, S.D. Yang and T. T. S. Kuo, Nucl. Phys. A462, 491 (1987).

[43] D. J. Rowe, 'Nuclear Collective Motions' (Methuen and Co. 1987).

[44] L. Coraggio, A. Covello, A. Gargano, N. Itako, T.T.S. Kuo, Prog. Part. Nucl. Phys.A805, 424(2008).

[45] H. Dong, T.T.S. Kuo and R. Machleidt, Phys. Rev. C 80, 065803 (2009).

[46] H.A. Bethe, Ann. Rev. Nucl. Sci. 21, 93 (1971).

[47] R. Machleidt, Adv. Nucl. Phys. 19, 189-376 (1989).

[48] J.W. Holt and G.E. Brown, p.239 in Hans Bethe and His Physics (World Scientific, July 2006, editted by G.E. Brown and C.-H. Lee).

[49] J.D. Holt, T.T.S. Kuo, G.E. Brown and S.K. Bogner, Nucl. Phys. A733, 152(2004).

[50] A. Fetter and J.D. Walecka, 'Quantum Theory of ManyParticle Systems' (MacGraw Hill Book Co. 1971).

[51] R.K. Su, S.D. Yang and T.T.S. Kuo, Phys. Rev. C35, 1539(1987). 